\title{
A NOTE ON FREE INVERSE SEMIGROUPS
}

\author{
by L. O'CARROLL
}

(Received 24th October 1972, revised 8th June 1973)

Recently Scheiblich (7) and Munn (3), amongst others, have given explicit constructions for $F I_{A}$, the free inverse semigroup on a non-empty set $A$. Further, Reilly (5) has investigated the free inverse subsemigroups of $F I_{A}$. In this note we generalise two of Reilly's lesser results, and also characterise the surjective endomorphisms of $F I_{A}$. The latter enables us to determine the group of automorphisms of $F I_{A}$, and to show that if $A$ is finite then $F I_{A}$ is Hopfian (a result proved independently by Munn (3)). Finally, we give an alternative proof of Reilly's main theorem, which uses Munn's theory of birooted trees.

Any results on free inverse semigroups which follow easily from (3), (7) are stated without proof. For the basic theory and notation of inverse semigroups see (1).

\section{1}

Let $S$ be an inverse semigroup with semilattice of idempotents $E$. Recall, (8), that the natural partial order $\leqq s$ on $S$ (usually denoted merely by $\leqq$ ) is defined as follows:

or equivalently,

$$
x \leqq y \text { iff } x=e y \text { for some } e \in E,
$$$$
x \leqq y \text { iff } x=y f \text { for some } f \in E \text {; }
$$

and that $\rho(S)=\{(x, y) \in S \times S \mid z \leqq x, z \leqq y$ for some $z \in S\}$ is the minimum group congruence on $S$.

Throughout this paper, let $A$ be a non-empty set and let $F I_{A}$ together with the canonical embedding, here denoted by $d: A \rightarrow F I_{A}$, be presented as in (3). In the notation of (3), for all $u, v \in F,[u] \geqq[v]$ iff $T(u) \subseteq T(v), \alpha(u)=\alpha(v)$ and $\beta(u)=\beta(v)$.

The inverse semigroup $S$ is said to be proper, (6), if $E \rho(S)=E$; in particular, $F I_{A}$ is proper. Moreover, $F I_{A} / \rho\left(F I_{A}\right)$ together with the canonical embedding $d \rho\left(F I_{A}\right)^{\natural}: A \rightarrow F I_{A} / \rho\left(F I_{A}\right)$ is " the " free group $F G_{A}$ on the set $A$.

Recall also, (9), that $F I_{A}^{1}$ is an $F$-inverse semigroup, in the terminology of (4). Scheiblich's theory (7) has an obvious interpretation in terms of $F$-inverse semigroups. Moreover, the set of maximal elements of $F I_{A}^{1}$ is in $1-1$ correspondence with the underlying set of the free group on $A$. This correspondence can be visualised in different ways, depending on whether one works with Scheiblich's theory (7) or with Munn's (3).

Let $U$ be an inverse subsemigroup of an inverse semigroup $S$. Then

$$
\rho(U) \subseteq \rho(S) \cap(U \times U)
$$

E.M.S. $-19 / 1-B$ 
so that a homomorphism $\alpha: U / \rho(U) \rightarrow S / \rho(S)$ is induced, given by:

$$
\text { for each } u \in U, \quad(u \rho(U))) \alpha=u \rho(S) \text {. }
$$

Proposition 1. Let $S$ be a proper inverse semigroup, and let $U$ be an inverse subsemigroup of $S$. Then

$$
\rho(U)=\rho(S) \cap(U \times U) .
$$

Proof. Let $(x, y) \in \rho(S) \cap(U \times U)$, and consider the element $x y^{-1} y$ of $U$.

Now $x y^{-1} \in U \cap E \rho(S)=U \cap E$, since $S$ is proper. Moreover, $y^{-1} y \in U \cap E$. Hence $x y^{-1} y=x \cdot y^{-1} y \leqq_{U} x$, and $x y^{-1} y=x y^{-1} \cdot y \leqq_{U} y$, so that $(x, y) \in \rho(U)$.

On the other hand, it is always the case that $\rho(U) \subseteq \rho(S) \cap(U \times U)$, and the result follows.

Corollary. Following the hypotheses of Proposition 1, the homomorphism $\alpha$ defined by (1) is injective. Each $\rho(S)$-class contains at most one $\rho(U)$-class.

It is easily seen that the hypothesis that $S$ be proper is essential in Proposition 1.

We now consider the case where the proper inverse semigroup $S$ is, in fact, $F I_{A}$. The induced homomorphism $\alpha$ then has codomain $F I_{A} / \rho(F I)_{A}=F G_{A}$. We deduce immediately:

Proposition 2. Let $U$ be an inverse subsemigroup of $F I_{A}$. Then

is injective.

$$
\alpha: U / \rho(U) \rightarrow F G_{A}
$$

A subset $K$ of an inverse semigroup [a group] $S$ is called a set of free generators for the inverse subsemigroup [the subgroup] $U$ of $S$ it generates if, given any mapping $g$ of $K$ into an inverse semigroup [a group] $T$, there exists a unique homomorphism $h$ of $U$ into $T$ such that $g=i h$, where $i: K \rightarrow U$ is the inclusion map.

We now state the main result of this section.

Theorem 1. In Proposition 2, suppose further that $U$ has a set of free generators $K$. Let $i: K \rightarrow U$ be the inclusion map, let $\gamma=i \rho(U)^{\natural} \alpha$, and let $H=(U / \rho(U)) \alpha$. Then $H \approx U / \rho(U),|K|=|K \gamma|$, and $K \gamma$ is a set of free generators for the group $H$.

Proof. By Proposition 2, $\alpha$ is injective and so $H \approx U / \rho(U)$. Clearly $K i \rho(U)^{\natural}$ is a set of free generators for the group $U / \rho(U)$. As remarked above, $i \rho(U)^{\natural}$ is the canonical embedding of $K$ in the group $U / \rho(U)$, and the result follows.

Theorem 1 can be paraphrased:

Let $A$ and $K$ be non-empty sets. If $F I_{A}$ contains a copy of $F I_{K}$, then $F G_{A}$ contains a copy of $F G_{K}$.

Proposition 2.8 of (5) now follows immediately. 
Suppose the hypotheses of Theorem 1 hold. Then in the light of Theorem 1 and the Corollary to Proposition $1, K$ is a transversal of the set of congruence classes $K \rho\left(F I_{A}\right)$, where $K \rho\left(F I_{A}\right)$ is a set of free generators for the subgroup $U \rho\left(F I_{A}\right)=(U / \rho(U)) \alpha$ of $F G_{A}$. In particular (5), Proposition 2.6 considers such transversals which moreover consist of maximal elements of $F I_{A}$.

For the final part of this section, the reader is referred to (2), Section 7.2.

Taking the converse situation, let $H$ be a non-trivial subgroup of $F G_{A}$, and let $N$ be a Schreier system of left coset representatives of $H$. Suppose $W$ is the corresponding set of Schreier generators of $H$-the $g s \phi(g s)^{-1}$ which are not equal to 1 , in the terminology of (2), Section 7.2. Then $W$ is a set of free generators for the group $H$, the elements of $W$ are reduced as written, and $W$ possesses central significant factors, namely the $s$ 's. Further, an element $g s \phi(g s)^{-1}$ of $W$ is clearly uniquely determined by its initial segment $g s$. Thus the proof of (5), Corollary 2.7, shows that $K=\{(I(w), w) \mid w \in W\}$-in the terminology of (5)-is a set of free generators for the inverse subsemigroup of $F I_{A}$ it generates; moreover, $|W|=|K|$.

In the terminology of (5), if $(R, r)$ and $(S, s)$ are any elements of $F I_{A}$, then $(R, r) \rho(S, s)$ iff $r=s$, while $(R, r) \leqq(S, s)$ iff $r=s$ and $S \subseteq R$. Thus $K$ in the preceding paragraph is a transversal of $\rho\left(F I_{A}\right)$-classes consisting of maximal elements of $F I_{A}$. Note also, given non-empty sets $B$ and $C$, that

$$
F I_{B} \approx F I_{C} \text { iff }|B|=|C|
$$

since the set of maximal idempotents $\left\{x x^{-1} \mid x \in A d \cup(A d)^{-1}\right\}$ of $F I_{A}$ has cardinality $2|A|$.

Thus the above can be paraphrased:

Let $A$ and $K$ be non-empty sets. If $F G_{A}$ contains a copy of $F G_{K}$, then $F I_{A}$ contains a copy of $F I_{K}$.

2

For convenience, let $\bar{A}=A d \cup(A d)^{-1}$. This notation will be used for the rest of this paper.

Lemma 1. Let $a \in \bar{A}$. Then $F I_{A} \mid\left\{a, a^{-1}\right\}$ is an inverse subsemigroup of $F I_{A}$.

Proof. This is obvious.

Suppose $\tau: A d \rightarrow F I_{A}$ is a map. We define an extension $\tau^{*}$ of $\tau$ to all of $\bar{A}$ as follows:

The domain of $\tau^{*}$ is $\vec{A}, \tau^{*} \mid A d=\tau$, and for each $x \in A d,\left(x^{-1}\right) \tau^{*}=(x \tau)^{-1}$.

Proposition 3. (i) Let $\delta$ be a surjective endomorphism of $F I_{A}$. Then $\bar{A} \subseteq \bar{A} \delta$.

(ii) Conversely, let $\tau: A d \rightarrow F I_{A}$ be a map such that $\bar{A} \subseteq \bar{A} \tau *$. Then $\tau$ can be extended to a unique endomorphism $\bar{\tau}$ of $F I_{A}$, and $\bar{\tau}$ is surjective. 
Proof. (i) Suppose there exists $a \in \bar{A} \backslash \bar{A} \delta$. Then $a^{-1} \in \bar{A}$, and if $a^{-1}=x \delta$ for some $x \in \bar{A}$ then $a=x^{-1} \delta$, where $x^{-1} \in \bar{A}$. Hence $a^{-1} \in \bar{A} \mid \bar{A} \delta$. It follows that $\bar{A} \delta \subseteq F I_{A} \mid\left\{a, a^{-1}\right\}$, which by Lemma 1 is an inverse subsemigroup of $F I_{A}$. But $\left(F I_{A}\right) \delta$ is the inverse subsemigroup of $F I_{A}$ which is generated by $\bar{A} \delta$, so that $\left(F I_{A}\right) \delta \subseteq F I_{A} \mid\left\{a, a^{-1}\right\}$, which is a contradiction.

(ii) Since $A d$ is a set of free generators for $F I_{A}, \tau$ can be extended in a unique manner to an endomorphism $\bar{\tau}$ of $F I_{A}$. Moreover, $\tau^{*}=\bar{\tau} \mid \bar{A}$ and it follows that $\bar{A} \subseteq\left(F I_{A}\right) \bar{\tau}$. Since $\bar{A}$ generates $F I_{A}$, we deduce that $\bar{\tau}$ is indeed surjective.

Let Aut $F I_{A}$ denote the group of automorphisms of $F I_{A}$. Given a nonempty set $X$, let $S_{X}$ denote the symmetric group on $X$.

Now follows the main result of this section:

Theorem 2. Let $A$ be a non-empty set, and let $A^{\prime}$ be a set disjoint from $A$ such that there exists a bijection $j: A \rightarrow A^{\prime}$ from $A$ on to $A^{\prime}$. Extend $j$ to a permutation $j^{*}$ on $A \cup A^{\prime}$ as follows:

Then

$$
\text { for each } x \in A\left[x \in A^{\prime}\right], \quad x j^{*}=x j\left[x j^{*}=(x) j^{-1}\right] .
$$

$$
\text { Aut } F I_{A} \approx\left\{\psi \in S_{A \cup A^{*}} \mid \psi j^{*}=j^{*} \psi\right\} \text {. }
$$

Proof. Define the permutation $t$ on $\bar{A}$ as follows:

$$
\text { for each } x \in \bar{A}, x t=x^{-1} \text {. }
$$

Let $V=\left\{\psi \in S_{\bar{A}} \mid \psi t=t \psi\right\}$, and let $T$ denote the right-hand side of (2). Then it is easily checked that $V$ and $T$ are groups, which are seen to be isomorphic when one identifies $j^{*}$ and $t$. It suffices therefore to prove that Aut $F I_{A} \approx V$.

Consider $\theta \in$ Aut $F I_{A}$; then $\theta^{-1} \in$ Aut $F I_{A}$, so that $\theta$ and $\theta^{-1}$ are, in particular, surjective endomorphisms of $F_{A_{A}}$. By Proposition 3, therefore, $\bar{A} \subseteq \bar{A} \theta$ and $\bar{A} \subseteq \bar{A} \theta^{-1}$. Hence $\bar{A} \theta=\bar{A}$, so that $\theta \mid \bar{A} \in S_{\bar{A}}$ and in fact $\theta \mid \bar{A} \in V$.

Define the map $k$ on Aut $F I_{A}$ as follows:

$$
\text { for each } \theta \in \text { Aut } F I_{A}, \quad k: \theta \rightarrow \theta \mid \bar{A} .
$$

From the preceding discussion it is clear that $k$ maps into $V$, and that $k$ is a homomorphism. Since $\bar{A}$ generates $F I_{A}$, we deduce that $k$ is injective.

Conversely, given $\psi \in V$ let $\tau=\psi \mid A d$. Then $\psi=\tau^{*}$. By Proposition 3, $\tau$ can be extended uniquely to a surjective endomorphism $\bar{\tau}$ of $F I_{A}$; clearly $\psi=\bar{\tau} \mid \bar{A}$. Similarly $\psi^{-1}$, which is in $V$, can be extended uniquely to a surjective endomorphism $\bar{\tau}^{\prime}$ of $F I_{A}$. Now $\bar{\tau} \bar{\tau}^{\prime} \mid \bar{A}$ and $\bar{\tau}^{\prime} \bar{\tau} \mid \bar{A}$ are both the identity map on $\bar{A}$. Since $\bar{A}$ generates $F I_{A}$, it follows that $\bar{\tau} \bar{\tau}^{\prime}$ and $\bar{\tau}^{\prime} \bar{\tau}$ are both the identity automorphism on $F I_{A}$. Hence $\bar{\tau}$ and $\bar{\tau}^{\prime}$ are mutually inverse automorphisms on $F I_{A}$, so that $k$ is surjective.

Corollary. Let $A$ be a non-empty finite set, with $n$ elements say, and let $B$ be the set of integers $r$ such that $-n \leqq r \leqq n$. Then

$$
\text { Aut } F I_{A} \approx\left\{\psi \in S_{B} \mid(-x) \psi=-(x \psi) \text { for all } x \in B\right\},
$$

and has $2^{n}(n !)$ elements. 
Proof. We need only note that if $\psi$ is an element of the right-hand side of (3), then $\psi(0)=0$ and $\psi$ is defined by its action on $\{1,2, \ldots, n\}$.

Proposition 4. If $A$ is finite, then $F I_{A}$ is Hopfian; that is to say, any surjective endomorphism $\theta$ of $F I_{A}$ is in fact an automorphism.

Proof. By Proposition $3, \bar{A} \subseteq \bar{A} \theta$. Since $\bar{A}$ is finite, we deduce that $\bar{A}=\bar{A} \theta$ and that $\theta \mid \bar{A}$ is injective. It follows, as in the proof of Theorem 2 , that $\theta$ is an automorphism.

This last result was proved independently by Munn (3).

3

In his main theorem, (5, Theorem 2.2), Reilly gives a necessary and sufficient condition that a subset of an inverse semigroup $S$ is a set of free generators for the inverse subsemigroup of $S$ it generates. In this final section, we use Munn's theory of birooted trees (3) to give an alternative proof of the sufficiency of Reilly's condition, a proof which we hope renders the condition conceptually clear. The necessity of the condition is almost immediate, especially if one again uses Munn's theory. But first we introduce some notation.

An element $t$ of $F I_{A}$ is said to be a reduced word in $\bar{A}$ if $t=x_{1} \ldots x_{n}$ for some $x_{1}, \ldots, x_{n} \in \bar{A}$ such that $x_{i} \neq x_{i+1}^{-1}, 1 \leqq i \leqq n-1$; in this case, the expression for $t$ is unique and $x_{1}\left[x_{n}\right]$ is called the first [last] letter in $t$. The reduced words $t_{i}=x_{1} \ldots x_{i}, 1 \leqq i \leqq n$, are called the initial segments of $t$; these are again unique.

Theorem 3. (Reilly). Let $K$ be a subset of an inverse semigroup $S$ such that $K \cap K^{-1}=\square$. Then $K$ is a set of free generators for the inverse subsemigroup $U$ of $S$ it generates iff the following condition is satisfied:

If $k \in K \cup K^{-1}$ and if $k k^{-1} \geqq \prod_{1}^{n} w_{i} w_{i}^{-1}$, where $w_{i}=k_{i 1} \ldots k_{i n(i)}$ for some $k_{i j} \in K \cup K^{-1}$ such that $k_{i j} \neq k_{i j+1}^{-1}$ for $1 \leqq j \leqq n(i)-1,1 \leqq i \leqq n$, then $k=k_{i 1}$ for some $i$.

Proof. (Sufficiency). Let $\theta: A \rightarrow K$ be a bijection of some set $A$ on to $K$. Then $\theta$ determines a unique epimorphism of $F I_{A}$ on to $U$, which we also denote by $\theta$. As remarked in (5), clearly $K$ is a set of free generators for $U$ iff $\theta$ is an isomorphism. Let $E$ denote the semilattice of idempotents of $F I_{A}$, and let $\sigma=\left(\theta \circ \theta^{-1}\right) \cap(E \times E)$.

Suppose, therefore, that $\theta$ is not an isomorphism. Following the remarks after Lemma 2.3 of (5), this is equivalent to supposing that $\sigma$ is not the identity congruence on $E$. Now $E$ satisfies the ascending chain condition, and the $\sigma$-classes are convex subsemilattices of $E$. Hence we may suppose that $e \theta=f \theta$ for some $e, f \in E$ such that $e$ covers $f$; that is to say, $e>f$ and if $e \geqq g>f$ for some $g \in E$, then $e=g$. Extend $\theta$ to an epimorphism, also denoted by $\theta$, of $F I_{A}^{1}$ on to $U^{1}$. 
Since $e$ covers $f$, with the notation of (3), we have that

$$
e=(T, \alpha, \alpha) \phi, f=\left(T^{\prime}, \alpha, \alpha\right) \phi,
$$

where $T^{\prime}$ is obtained from $T$ by the addition of exactly one extra vertex, $\gamma$ say, to $T$ and a corresponding edge $\beta \gamma$, for some vertex $\beta \in T$. Let $x$ be the label on $\overrightarrow{\beta \gamma}$ and let $w \in F I_{A}^{1}$ be the (reduced) word corresponding to the shortest path on $T$ from $\alpha$ to $\beta$ (where $w=1$ if $\alpha=\beta$ ). Now let

$$
e^{*}=w^{-1} e w, f^{*}=w^{-1} f w .
$$

Then $e^{*}=(T, \beta, \beta) \phi, f^{*}=\left(T^{\prime}, \beta, \beta\right) \phi, e^{*} \theta=f^{*} \theta$ and $e^{*}$ covers $f^{*}$. Further, $e^{*} x x^{-1}=f^{*}$.

Let $k=x \theta \in K \cup K^{-1}$. Then

$$
e^{*} \theta=f^{*} \theta=\left(e^{*} x x^{-1}\right) \theta=e^{*} \theta \cdot k k^{-1},
$$

so that $k k^{-1} \geqq e^{*} \theta$. Starting from $\beta$ and going in turn to each of the various extremities of $T$ by the shortest path gives rise to reduced words in $\bar{A}, z_{1}, \ldots, z_{n}$ say, where $z_{i}=x_{i 1} \ldots x_{i n(i)}$ for unique $x_{i j} \in \bar{A}$ such that

Thus

$$
x_{i j} \neq x_{i j+1}^{-1}, 1 \leqq j \leqq n(i)-1, \quad 1 \leqq i \leqq n .
$$

$$
e^{*}=(T, \beta, \beta) \phi=\prod_{1}^{n} z_{i} z_{i}^{-1}
$$

No oriented edge in $T$ emanating from $\beta$ is labelled by $x$, so that $x \neq x_{i 1}$, $1 \leqq i \leqq n$.

Thus

$$
k k^{-1} \geqq e^{*} \theta=\left(\prod_{1}^{n} z_{i} z_{i}^{-1}\right) \theta=\prod_{1}^{n} z_{i} \theta\left(z_{i} \theta\right)^{-1},
$$

where $z_{i} \theta=x_{i 1} \theta \ldots \ldots x_{i n(i)} \theta, 1 \leqq i \leqq n$. Moreover, $\theta$ is a bijection of $\bar{A}$ on to $K \cup K^{-1}$, so that for each $i, 1 \leqq i \leqq n, x_{i j} \theta \neq\left(x_{i j+1} \theta\right)^{-1}$ for $1 \leqq j \leqq n(i)-1$, and $k=x \theta \neq x_{i 1} \theta$.

The result follows.

I would like to thank the referee for his helpful suggestions, and in particular for his extremely neat formulation of part of the proof of Theorem 3 .

\section{REFERENCES}

(1) A. H. Clifford and G. B. Preston, The Algebraic Theory of Semigroups (Math. Surveys No. 7, Amer. Math. Soc., Providence, Vol. I, 1961 and Vol. II, 1967).

(2) M. HALL, Theory of Groups (Macmillan, 1959).

(3) W. D. Munn, Free inverse semigroups, Semigroup Forum, 5 (1973), 262-269. 
(4) R. McFadden and L. O'Carroll, F-inverse semigroups, Proc. London Math. Soc. (3) 22 (1971), 652-666.

(5) N. R. ReILLy, Free generators in free inverse semigroups, Bull. Austral. Math. Soc. 7 (1972), 407-424.

(6) T. SAIto, Proper ordered inverse semigroups, Pacific J. Math. 15 (1965), 649-666.

(7) H. E. Scheiblich, Free inverse semigroups, Semigroup Forum, 4 (1972), 351-359.

(8) V. V. VAGNer, Theory of generalised heaps and generalised groups, Mat. Sb. (NS) 32 (1953), 545-632. (Russian.)

(9) V. V. VAGNER, Generalised heaps and generalised groups with a transitive compatibility relation, Učen. Zap. Saratov. Gos. Univ., meh.-matem., 70 (1961), 25-39. (Russian.)

Mathematical Institute

Chambers Street

EDINBURGH EH1 $1 \mathrm{HZ}$ 